\title{
Empirical Study on Agglomeration Effect of Regional Big Data Industry
}

\author{
Junmeng Lu Mu Zhang
}

School of Finance, Guizhou University of Finance and Economics, Guiyang Guizhou 550025 , China

\begin{abstract}
Big data industry contains enormous economic value, its development will provide an incredible impetus to the future economic growth in our country. The agglomeration of an industry in a certain area can effectively increase the production efficiency and the output of the industry. This phenomenon is even more obvious in the manufacturing industry. In this paper, the location quotient is included in the Cobb-Douglas production function as a variable that reflects the agglomeration effect of big data industry. The double logarithmic panel data model is used to carry out empirical analysis to estimate the economic impact of regional agglomeration on the big data industry. According to the results of the research, location quotient have a significant positive effect on the output of the big data industry, that is, the agglomeration of the big data industry within a certain region can obviously promote the industrial development.
\end{abstract}

\section{Keywords}

big data; agglomeration effects; location quotient

\section{区域大数据产业集聚效应的实证研究}

\author{
卢俊萌 张目 \\ 贵州财经大学金融学院, 贵阳 550025, 中国
}

摘要: 大数据产业蕴含巨大经济价值, 其发展对我国以后经济增长可产生难以估量的推动作 用。一定区域内某一产业的集聚可有效提高提高该产业的生产效率从而增加产出，该现象在 制造业中更为显著。本文将区位熵作为反映大数据产业集聚效应的变量纳入柯布一道格拉斯 生产函数中, 通过构建双对数面板数据模型进行实证分析, 测算区域集聚效应对大数据产业 的经济影响。从研究结果可得, 区位熵对大数据产业产出具有显著正效应, 即一定区域内大 数据产业的集聚对产业发展具有明显的促进作用。

关键词: 大数据; 集聚效应; 区位摘 


\section{1. 引言}

近年来，“大数据”一词不断出 现在公众的视野中，成为继物联网、 云计算和移动互联网之后的又一个信 息技术热点。大数据主要是指在无法 可容忍的时间内，利用常用的 IT 技术 和软硬件对其感知、获取、管理、处 理和服务的数据集合 ${ }^{[1]}$ 。其主要有四个 特点, 分别是规模性(volume)、多样性 (variety)、高速性 (velocity) 和价值性

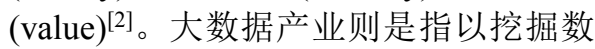
据分析服务为核心，一切与大数据的 产生与集聚、组织与管理、分析与发 现、应用与服务相关的经济活动集 合。涵盖了数字内容服务、物联网服 务、位置服务等信息服务; 数据中 心、宽带网络等基础设施服务; 智能 交通、互联网金融、智慧城市等应用 服务; 以及智能终端制造、电子元器 件制造等电子产品制造业 ${ }^{[3]}$ 。世界经 济论坛在 2012 年发布的《大数据、大 影响》中，从互联网、金融、教育、 医疗等多个行业领域指出大数据正在 世界经济带来巨大的影响和发展机遇 [4]。发达国家也纷纷加大对大数据产业 发展的支持, 将大数据纳入国家发展 战略。我国大数据产业亦呈良好发展 态势，在国家政策的大力推动下，贵 州、广东、上海和重庆等地方政府先 后出台大数据产业的相关发展规划纲 要 ${ }^{[5]}$ 。可见, 大数据隐含的巨大经济价 值和发展机遇已受到极大的重视, 发 展大数据产业无疑会给经济、科研、 互联网等产生巨大的推动作用。

产业集聚，指产业活动在一定地 理范围内的集中和聚积, 即产业内生 产活动、销售活动及为该种生产和销 售所服务的经济活动在一定区域内高 度集中的现象 ${ }^{[6]}$ 。源于新经济地理学有 关产业集聚的观点普遍认为, 某一产 业的集聚常使得该产业获得外部规模
经济, 使得相应的生产活动表现出规 模报酬递增的特性, 进而在经济增长 方面表现为产出增加及生产效率提高 [7]。但陈建军、胡晨光则认为, 产业集 聚未必存在规模报酬递增, 而是产业 集聚产生的索洛剩余递增既构成区域 产业集聚循环累积因果机制的集聚 力, 又构成扩散力。当一定区域内产 业集聚达到一定程度时, 会促使传统 产业索洛剩余递减, 导致传统产业向 该区域以外转移 ${ }^{[8]}$ 。王光玲、吴学花通 过测度中国制造业集聚水平对生产产 出的促进效果时发现, 中国制造业存 在显著的集聚效应 ${ }^{[9]}$ 。目前, 我国大数 据产业仍处于发展初期, 产业集聚程 度较低, 一定区域内的大数据产业集 聚应有助于提高生产效率、增加产 出。要着力发展大数据产业, 则需优 先发展支撑大数据产业的制造业，从 而为大数据产业后续发展奠定良好的 经济基础, 抢占大数据的制高点。

从根本上讲，规模经济反映生产 要素投入与产出之间的相关程度, 故 用于度量规模经济的传统生产函数分 析方法亦可用于度量产业集聚效应与 经济增长之间的关系 ${ }^{[10]}$ 。本文采用区 位熵法对产业集聚效应进行度量, 并 将区位熵作为生产函数的一个解释变 量, 通过面板数据模型分析其对大数 据产业的产出是否带来正效应。

\section{2. 区域大数据产业集聚效应的测度}

\section{1. 模型设计}

为实证考察集聚效应对大数据产 业产出是否带来正效应, 本文选用柯 布一道格拉斯生产函数作为分析函 数, 并将区位摘及出口完成作为一个 解释变量纳入函数中, 模型如下:

$$
Y=\mathrm{A} K^{\alpha} L^{\beta} C^{\gamma} J^{\lambda}
$$

其中, $Y$ 表示总产出, $K$ 表示资本 投入量, $L$ 表示劳动投入量, $C$ 表示出 
口情况, $J$ 则表示反映集聚水平的影响 因素。并选取大数据产业销售产值 （sales value, SV）为被解释变量, 选 取自年初累计完成投资（annual investment, AI ) 、出口完成 (completed export, CE)、全部从业 人员平均人数 ( average of all employees, AAE) 及区位摘（location quotient, LQ）作为解释变量, 同时为 减少模型的异方差性, 本文对公式（21) 取自然对数, 从而构建如下大数据 产业产出的集聚效应双对数面板数据 模型:

\section{$\ln S V_{\text {it }}=\beta_{0}+\beta_{1} \ln A I_{\text {it }}+\beta_{2} \ln C E_{\text {it }}+$ $\beta_{\mathrm{a}} \ln A A E_{\mathrm{it}}+\beta_{4} \ln L Q_{\mathrm{it}}+u_{\mathrm{it}}$}

其中, $u_{i t}$ 表示 “非主要因素” 的 影响、随机变化、观测误差和模型数 学形式设定偏差等各种因素对被解释 变量 $S V$ 的影响总和, 通常称为随机扰 动项; 常数 $\beta_{0}, \beta_{1}, \ldots \ldots, \beta_{3}$ 为待定参 数。模型中 $i$ 为第 $i$ 个地区 $(i=1,2, \cdots, 31) ; t$ 为第 $t$ 个年份 $(t=1,2, \cdots, 5)$; it 为第 $t$ 年份的第 $i$ 地区。统计分析软件采用 Stata 11 。

\section{2. 数据说明}

本文收集了 2011-2015 年我国 31 个省、自治区、直辖市的大数据产业 的变量数据, 因此, $i=1,2, \cdots, 31$, $t=1,2, \cdots, 5$ 。原始数据来源于 《中 国电子信息产业统计年鉴》各期。限 于篇幅, 原始数据略。由于数据来源 的局限性, 本文仅统计到大数据产业 销售产值（SV）、自年初累计完成投 资 $(\mathrm{AI})$ 、出口完成 $(\mathrm{CE})$ 的变量数 据。

全部从业人员平均人数 ( AAE) 因 2013-2015 年三个年份的《中国电子 信息产业统计年鉴》在全国范围内均 未对该变量进行统计, 数据缺失严 重, 故从《中国高技术产业年鉴》提
取该解释变量各个年份数据。根据 《中国电子信息产业统计年鉴》中的 地区划分标准, 我国东部地区包括: 北京、天津、上海、河北、辽宁、江 苏、浙江、福建、山东、广东、广西 和海南等 12 个省（市）; 中部地区包 括: 山西、内蒙古、吉林、黑龙江、 安徽、江西、河南、湖北、湖南等 9 个省（区）；西部地区包括：四川、 重庆、贵州、云南、陕西、甘肃、青 海、宁夏、新疆等 10 个省（区）。其 中, 年鉴未统计西藏地区 2011-2014 年 份的各变量数据, 该地区数据缺失严 重, 故将该地区从实证分析中剔除。

区位熵（LQ），也称地区专业化 指数, 常用于测度某地区生产结构中 的某个产业与全国水平相比较下其所

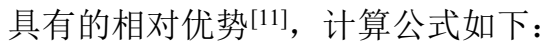

$$
L Q_{i t}=\frac{\mathrm{X}_{i t} / \sum_{t} X_{i t}}{\sum_{i} X_{i t} / \sum_{t} \sum_{i} X_{i t}}
$$

公式中, $i$ 为第 $i$ 个地区 $(i=1,2, \cdots, 31) ; t$ 为第 $t$ 年份; $X_{i t}$ 为第 $t$ 年份的第 $i$ 个地区的主营业务收 入。若 $L Q>1$, 则在该地区内该产业的 生产专业化水平高于全国水平，同时 该产业在该地区相对集中, 具有较高 集中度和规模经济特性; 若 $L Q<1$, 则 表明该地区内该产业的集聚程度低于 全国水平; 若 $L Q=1$, 则表明该地区内 该产业的集聚程度与全国水平持平。

\section{3. 大数据产业集聚与产业经济增长的 实证分析}

\subsection{Hausman 检验}

通过 Hausman 检验, 判断选择固 定效应模型（fixed-effects model）还是 随机效应模型 ( random-effects model）。Hausman 统计量为:

$\mathrm{H}=(\mathrm{b}-\mathrm{B})^{\prime}[\operatorname{Var}(\mathrm{b})-\operatorname{Var}(\mathrm{B})]-1(\mathrm{~b}-\mathrm{B}) \sim \chi^{2}(\mathrm{k})$ 
Hausman 统计量服从自由度为 $\mathrm{k}$ 的 $\chi^{2}$ 分布。当 $\mathrm{H}$ 大于一定显著水平的 临界值时, 就可以认为模型中存在固 定效应，从而选用固定效应模型，否 则选用随机效应模型。根据 2011-2015 年我国 31 个省、自治区、直辖市大数 据产业数据（其中，西藏地区 20112014 年数据缺失），利用 Stata11.2 软 件进行 Hausman 检验, 结果如表 1 所 示。

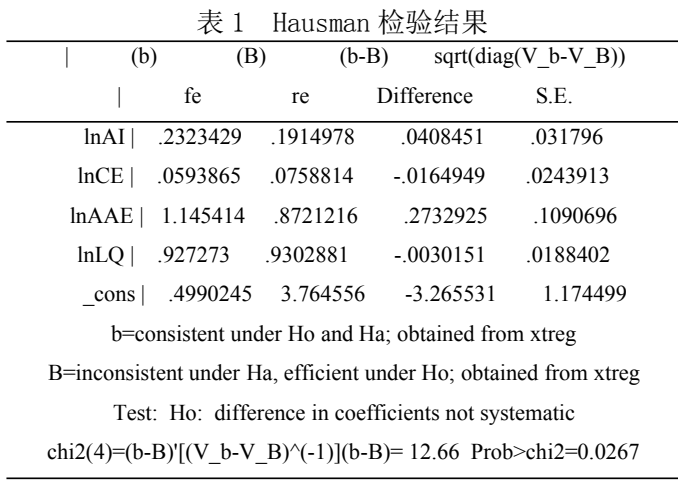

\section{由表 1 可以看出,} Prob $>$ chi $2=0.0267$, Hausman 检验在 $5 \%$ 的显著性水平上显著, 可以拒绝原 假设, 即相对于运用随机效应模型进 行估计, 对收集的样本数据采用固定 效应模型进行估计更为合适。

\section{2. 固定效应模型回归结果}

根据 2011-2015 年我国 31 个省、 自治区、直辖市大数据产业原始数 据, 利用 Stata11 软件对式 (2-2) 进行 参数估计, 估计结果无不显著变量, 得出的固定效应模型回归结果如表 2 所示。

表 2 固定效应模型回归结果

\begin{tabular}{|c|c|c|c|c|c|}
\hline $\operatorname{lnSV} \mid$ & Coef. & Std. Err. & $\mathrm{t}$ & $\mathrm{P}>|\mathrm{t}|$ & [95\% Conf. Interval] \\
\hline $\operatorname{lnAI} \mid$ & .2323429 & .0529978 & 4.38 & 0.000 & .3373117 \\
\hline $\operatorname{lnCE} \mid$ & .0593865 & .0397712 & 1.49 & 0.138 & -.0193853 \\
\hline $\ln \mathrm{AAE}$ & | 1.145414 & .1205454 & 9.50 & 0.000 & .9066588 \\
\hline $\operatorname{lnLQ} \mid$ & $\begin{array}{l}\mid .927273 \\
\end{array}$ & .039939 & 23.22 & 0.000 & .8481689 \\
\hline _cons | & | . .4990245 & 1.213662 & 0.41 & $\begin{array}{r}0.682 \\
744714\end{array}$ & -1.9047862 .902835 \\
\hline
\end{tabular}

续表 2 固定效应模型回归结果 sigma_e | .167853

rho $\quad .92134211$ (fraction of variance due to $u_{-} i$ )

F test that all u_i=0: $F(29,116)=12.41 \quad$ Prob $>F=0.0000$

R-sq: overall $=0.9763 \quad \operatorname{corr}(\mathrm{u}$ i, $\mathrm{Xb})=-0.8629 \quad \mathrm{~F}(4,116)=267.68$ Prob $>$ F $=0.0000$

由表 2 可得，模型中个体效应和 随机干扰项的方差估计值分别为 sigma $\mathrm{u}=0.5744714$ 和 $\operatorname{sigma} \mathrm{e}=0.167853$, 二 者之间的相关关系 rho 不等于零，表明 面板水平方差组分是重要的。且面板 估计（panel estimator）与联合估计

( pooled estimator) 是相同的。 $\mathrm{F}(29,116)=12.41, \quad \operatorname{Prob}>\mathrm{F}=0.0000$, 表 明在 $1 \%$ 的显著性水平上固定效应是显 著的。模型拟合优度为 0.9763 , 即模 型的解释能力为 $97.63 \%$ 。但解释变量 出口完成 (CE) 不显著, 将该变量剔 除, 再次进行固定效应模型分析, 模 型回归结果如表 3 所示。

表 3 固定效应模型回归结果

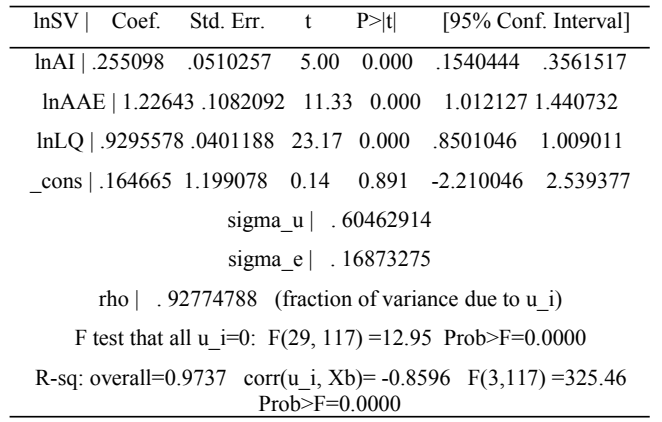

由表 2 可得, 模型中个体效应和 随机干扰项的方差估计值分别为 sigma $\mathrm{u}=0 . \quad 60462914 \quad$ 和 sigma $\mathrm{e}=0.16873275$ ，二者之间的相关关系

(面板水平方差组分对总方差的贡献 比例 ) $\mathrm{rho}=0.92774788$, 即 rho 不等于 零, 表明面板水平方差组分是重要 的。且面板估计 (panel estimator) 与 联合估计（pooled estimator）是相同 的 $。 \quad F(29,117)=12.95$, Prob $>F=0.0000$, 表明在 $1 \%$ 的显著性 水平上固定效应是显著的。模型的拟 合优度为 0.9737 , 说明模型的解释能 
力为 $97.37 \%$, 即自年初累计完成投资 (AI) 、全部从业人员平均人数 (AAE) 及区位熵（LQ）能够对大数 据产业销售产值（SV）的 97.63\%作出 解释。此外, corr(u_i, Xb) $=-0.8596$, 表明不可观测的个体效应与解释变量 的相关性较高, 有固定效应。 $F(3,117)=325.46, \quad$ Prob $>F=0.0000$ ，表 明模型估计系数在 $1 \%$ 的显著性水平上 总体显著。从变量的估计系数来看, 自年初累计完成投资（AI）、全部从 业人员平均人数（AAE）、区位摘 (LQ) 均在 $1 \%$ 的显著性水平上对大 数据产业销售产值（SV）存在显著的 正向影响, 弹性分别为 $0.2551 、 1.2264$ 和 0.9296 。

\section{4. 结论与讨论}

本文采用柯布一道格拉斯生产函 数对电子信息产业产出进行度量, 通 过构建双对数面板数据模型, 开创性 地将出口情况及产业集聚效应作为产 业产出的影响因素纳入模型中进行分 析。

实证分析结果得出, 区位摘 （LQ）在 $1 \%$ 的显著性水平上对全国 大数据产业销售产值 (SV) 的弹性系 数为 0.9296 。自年初累计完成投资 ( AI ) 与全部从业人员平均人数 （AAE）在 1\%显著性水平上的弹性系 数仅为 0.2551 和 1.2264 , 而出口完成

(CE) 对大数据产业销售产值 ( SV) 不存在显著影响。分析结果表明, 通 过一定区域内的大数据产业集聚可有 效刺激产业的经济增长。

本文通过实证分析得出大数据产 业的集聚效应与其产业产出存在正相 关关系，利用产业集聚效应的规模报 酬递增特性, 其发展可有效提高和扩 大大数据产业的发展速度和规模。金 显、陈钊等人认为, 影响产业集聚效 应的因素主要有经济开放、地理位
置、市场容量、城市化、基础设施完 善程度及政府作用等 ${ }^{[12], ~}$ 故提高我国 的大数据产业集聚效应可从上述几个 方面着手, 从而推动大数据产业乃至 我国经济的发展。

以本文研究结果可为我国加快发 展大数据产业和推动经济发展的作参 考。由于部分数据统计不充分、行业 资料获取存在一定困难及编者能力有 限, 使得模型分析结果的有效性相对 较参差, 对实证分析结果产生一定影 响, 后续研究可在这些方面作出进一 步的改善。

\section{致谢}

基金项目：本文获得 2017 年度第 二批贵州省基础研究计划（软科学类 别）项目（黔科合基础（2017）15161）: 贵州大数据产业、生态文明建设 与金融集聚耦合协调发展研究; 2017 年度贵州财经大学校级科研项目（青 年项目）“贵州大数据产业集聚效应 及金融支持体系研究” 资助。

\section{参考文献}

[1] 李国杰,程学旗. 大数据研究:未来 科技及经济社会发展的重大战略 领域一大数据的研究现状与科 学思考 [J]. ,2012,27(06):647-657.

[2] 孟小峰,慈祥. 大数据管理: 概念、 技术与挑战 [J]. ,2013,50(01):146169.

[3] 省人民政府印发《关于加快大数 据产业发展应用若干政策的意 见》、《贵州省大数据产业发展 应用规划纲要(2014-2020 年)》的 通知 [J]. 2014,(03):9-21.

[4] Consulting V W. Big Data, Big Impact: New Possibilities for International Development[J]. 2012. 
[5] 陈立枢. 中国大数据产业发展态势 及 政 策 体 系 构 建 [J]. ,2015,31(06):144-147.

[6] 徐康宁. 产业聚集形成的原因和影 响的研究[D].复旦大学, 2003 .

[7] 梁华峰.澳门服务产业集聚效应实 证 分 析 $[\mathrm{J}]$. 经 济 地 理,2011,31(02):266-270.

[8] 陈建军,胡晨光. 产业集聚的集聚 效应一以长江三角洲次区域为 例的理论和实证分析 [J]. 管理世 界,2008,(06):68-83.

[9] 王光玲,吴学花. 中国制造业集聚 对生产的贡献度检验 $[\mathrm{J}]$. 统计与决 策,2008,(17):77-79.

[10] 刘长全. 集聚经济测度方法前沿综 述 [J]. 统计研究,2006,(03):38-41.

[11] 陈迅,陈军. 产业集聚效应与区域 经济增长关系实证分析 [J]. 华东经 济管理,2011,25(02):33-35.

[12] 金显,陈钊,陆铭. 中国的地区工业 集聚:经济地理、新经济地理与经 济政策[J]. 经济研究,2006,(04):7989. 